Notfall Rettungsmed 2015 · 18:232

DOI 10.1007/s10049-015-0027-7

Online publiziert: 7. Mai 2015

(c) Springer-Verlag Berlin Heidelberg 2015

H.-C. Schaub

Schulaufsicht für das Fach Gesundheit, Rettungsassistent, Remseck am Neckar, Deutschland

\title{
Kommentar zu: Laienreanimation nach kürzlich durchgeführtem Erste-Hilfe-Kurs
}

\section{Zum Beitrag \\ Winkler BE, Henssler J, Piepho T et al (2014) \\ Laienreanimation nach kürzlich durchgeführ- tem Erste-Hilfe-Kurs. Notfall + Rettungsmedi- zin 17:684-689}

Der Artikel beschreibt sehr gut Defizite im Ergebnis der Laienausbildung im Bereich der Reanimation. Die Schlussfolgerungen jedoch erscheinen mir unvollständig, da ich eine andere Erklärung für die beobachteten Ergebnisse habe.

Im Rahmen einer Lehrkräfteweiterbildung (Lehrkräfte beruflicher Schulen, die z. B. Notfallmedizin in Berufsschulen unterrichten und dort auch Wiederbelebung trainieren) haben wir eine umfangreiche Simulationspuppe (Leardal QCPR) eingesetzt. Zum ersten Mal erhielten also Teilnehmende neben dem Feedback einer beobachtenden Person Daten über die messbare Leistung.

Es wurde deutlich, dass (auch geübte Beobachter) die tatsächlich erbachten Leistungen (v. a. im Bereich Beatmungsvolumen und Drucktiefe) nicht korrekt einschätzen konnten.

In Korrespondenz zu den Ergebnissen des Beitrags lässt das für mich nur folgende Schlussfolgerungen zu:

1. Ausbildungskräfte müssen Übungsphantome einsetzen, die über entsprechende Auswertungsoptionen verfügen. Derzeitig werden jedoch preisgünstige Phantome ohne solche Möglichkeiten in Erste-Hilfe-Kursen eingesetzt.

2. Ausbildungskräfte müssen in der Beobachtung und Rückmeldung trainiert werden. Die Gestaltung von Trainingsphasen ist (wie auch durch andere Untersuchungen mehr- fach bestätigt) pädagogisch-didaktisch häufig schlecht und findet gerade im Laienbereich unter Bedingungen statt, die nicht als lernförderlich zu bezeichnen sind.

3. Die Kursqualität im Bereich der Laienausbildung ist in vielen Fällen als mangelhaft zu bezeichnen. Die von den Autoren festgestellte Performanz spiegelt die nur bedingt geförderten Kompetenzen durch die Kurskonzepte und deren Umsetzung. CO-CPR wäre eine Inhaltsreduktion an falscher Stelle. Die Kurskonzepte beinhalten heute so viel thematischen Ballast, dass eine konsequente Ausrichtung auf die lebensrettenden Maßnahmen (insbesondere CPR) keiner Verkürzung des Inhalts an dieser Stelle bedarf.

Die Schlussfolgerung über Personen, die ehrenamtlich in Rettungsorganisationen tätig sind, bezieht sich auf die Untersuchungsgruppe der Autoren und hat damit die Einschränkung, dass es sich um Personen der gymnasialen Oberstufe handelt. Diese können keine umfangreiche fachlich fundiert Ausbildung durchlaufen (z. B. Rettungssanitäter) haben.

Mit freundlichen Grüßen Christoph Schaub

\section{Korrespondenzadresse}

\section{H.-C. Schaub}

Schulaufsicht für das Fach Gesundheit, Rettungsassistent

Bostonring 37/3, 71686 Remseck am Neckar mail@christophschaub.de 